\title{
Informed Consent und Shared Decision Making1 . Ein Überblick über medizinische und sozialwissenschaftliche Literatur
}

\author{
Von Gunnar Stollberg ${ }^{2}$
}

Zusammenfassung: Die Rollen des Konsumenten der Angebote im Gesundheits- und WellnessBereich, des Patienten im System der Krankenversorgung und des Bürgers eines Wohlfahrtsstaates überschneiden sich und sind im Zuge der Umstrukturierungen des „Gesundheitswesens“ Wandlungen unterworfen (so Kickbusch). Dass Konsumenten in ökonomischer und Bürger in politischer Hinsicht Entscheidungen fällen müssen, ohne dass diese wissenschaftlich ,abgedeckt“ sind, ist trivial. Weniger trivial scheint dies für die Patienten. Die erwähnten Umstrukturierungen bringen jedoch die Erwartung mit sich, dass auch Patienten an Entscheidungen zumindest beteiligt werden. Problematisch an dieser Erwartung scheint, dass Eigentümlichkeiten des medizinischen und des politischen Systems vermischt werden. Dies geschieht, indem die Gesellschaft als Gesundheitsgesellschaft definiert wird und mit dieser Definition Ansprüche an Individuen begründet werden, denen diese nicht unmittelbar, sondern nur durch Entwicklung von Gesundheitskompetenz (patient empowerment) entsprechen können. Daher will ich shared decision making als ,,modernen Mythos der Medizin“ vorstellen, als ein praktikables Schema, das ein Versprechen formuliert (Vgl. Vogd / Saake): Die Unsicherheit einer Krankheitssituation kann nicht unmittelbar durch medizinische Definition und Intervention, sondern soll durch Rekurs auf Partizipation entschärft werden.

\section{Einleitung}

Ilona Kickbusch hat in einem gesundheitswissenschaftlich wie gesundheitspolitisch einflussreichen Buch darauf hingewiesen, dass sich in der "Gesundheitsgesellschaft" verschiedene Rollen überschneiden: Eine Person ist auf dem Gesundheitsmarkt ein Konsument, im Gesundheitssystem (System der Krankenversorgung) ein Patient und in der Gesundheitsgesellschaft ein Bürger. Es komme im Zuge der Veränderungen in allen diesen Bereichen zu Änderungen, die stets neue Rollenverschiebungen mit sich brächten. „Wenn die Gesundheitsgesellschaft eines aktiven und kritischen Bürgers, Konsumenten und Patienten bedarf, dann müssen diese ein gebührendes $\mathrm{Ma} ß$ an Gesundheitskompetenz besitzen. Gesundheitskompetenz ist die Fähigkeit des Einzelnen im täglichen Leben Entscheidungen zu treffen, die sich positiv auf die Gesundheit auswirken“ (Kickbusch 2006: 69).

Diese Rollenüberschneidung ist an sich nicht verwunderlich, ist doch die moderne Gesellschaft eben durch eine Vielfalt von Rollen charakterisiert, die Personen einnehmen können und müssen. Problematisch scheint mir jedoch, dass Eigentümlichkeiten des medizinischen und des politischen Systems vermischt werden. Dies geschieht, indem die Gesellschaft als Gesundheitsgesellschaft ${ }^{3}$ definiert wird und mit dieser Definition Ansprüche an Individuen begründet werden, denen diese nicht unmittelbar, sondern nur durch Entwicklung von Gesundheitskompetenz (patient empowerment) entsprechen können. Angesichts der Demokratisierung des politischen Systems, die die Gleichheit der Staatsbürger betont und trotz aller

1) Die deutschen Übersetzungen „,informierte Einwilligung“ bzw. „partizipative Entscheidungsfindung" sind bisher wenig gebräuchlich und werden im wissenschaftlichen Kontext meist mit den englischen Begriffen erläutert.

2) Dem anonymen Gutachter / der Gutachterin sei für seine / ihre Kritik herzlich gedankt!

3) Diese Definition ist angesichts des hohen Werts, der Gesundheit und Wellness beigemessen wird, durchaus berechtigt. Sie konkurriert jedoch mit Begriffen wie Wissens-, Erlebnis-, Netzwerk- etc. gesellschaft und bleibt insofern perspektivisch beschränkt. 
Professionalisierungstendenzen, ${ }^{4}$ von denen bereits Max Weber sprach, keine formalisierten Anforderungen an die Expertenrolle ausgeprägt hat, erscheint das Arzt-Patient-Verhältnis ${ }^{5}$ als rückständig und anstößig. Ein Angleichungsprozess werde erforderlich, der wiederum auf Expertisierungstendenzen auf Seiten der Patienten rekurrieren könne. Informed consent (IC) und shared decision making (SDM) könnten diese Angleichung erleichtern. Zu dieser Partizipation seien jedoch Patienten nur begrenzt in der Lage. Sie müssten qualifiziert (empowered) werden. Dabei könne auf Expertisierungswünsche und -tendenzen der Patienten zurückgegriffen werden.

Ich werde das SDM-Konzept als einen modernen Mythos der Medizin (Vgl. Vogd / Saake 2008) vorstellen, d.h. als ein Schema, das ein Versprechen impliziert. SDM verspricht, das politisch anstößige Arzt-Patient-Verhältnis durch Partizipation zu demokratisieren.

Ich werde mit folgenden Schritten vorgehen: Zunächst werde ich SDM im Verhältnis zu anderen soziologischen Beschreibungen der Arzt-Patient-Beziehung näher umreißen: zum klassischen, als paternalistisch kritisierten Modell von Parsons und zum Modell des informed consent (IC). Dabei werde ich auf die Selbsthilfebewegung als Kontext der Kritik an Parsons eingehen und auf die korporatistische Unterfütterung der neuen Modelle in Deutschland. Zweitens werde ich die medizinsoziologische Literatur dahingehend befragen, ob sich das medizinische Laien- dem Expertenwissen angenähert hat. Drittens werde ich empirische Studien zum Bedürfnis ,der“ Patienten nach Information und Beteiligung resümieren. Abschließend werde ich Schlussfolgerungen zum Vertrauensbegriff und zu SDM als modernem Mythos der Medizin ziehen.

\section{Die Modelle des informed consent und des shared decision making}

Das SDM-Modell wurde in Kritik der als paternalistisch empfundenen Analyse der Arzt-Patient-Beziehung durch Parsons entwickelt. Dabei wurde das IC-Modell als Zwischenstufe konstruiert.

Parsons hatte die Situation des Patienten als durch Hilflosigkeit und Hilfsbedürftigkeit geprägt beschrieben. Kranke seien nicht befähigt, sich selbst zu helfen, bzw. sei das, was sie täten, nicht situationsadäquat. Ferner erschwere ihre Betroffenheit ein rationales Urteil. Komplementär dazu sei die Situation des Arztes beschaffen: er verfüge über wissenschaftliche medizinische Kenntnisse und sei insofern befähigt, zu helfen (Vgl. Parsons 1951; Stollberg 2001: 8).

Für die medizinische Praxis charakterisieren Charles, Gafni und Whelan (1999: 653) das IC-Modell wie folgt: Der Arzt gibt dem Patienten die Informationen, die für die therapeutische Entscheidung relevant sind. Der Patient erwägt diese Informationen, diskutiert sie gegebenenfalls mit anderen und fällt dann die Entscheidung (Vgl. auch Emanuel / Emanuel 1992).

Das IC-Modell kommt nicht aus einer sozialwissenschaftlichen Analyse der Arzt-PatientBeziehung, sondern aus der medizinischen Forschung. Dort wurde seit dem späten 19. Jahrhundert eine Informationspflicht der Mediziner gegenüber den Untersuchungspersonen institutionalisiert (Vgl. Noack 2004; Sabisch 2007). Diese Pflicht der medizinischen Forscher, ihre Versuchspersonen zu informieren, wurde im weiteren Verlauf zu einer Pflicht, deren Zustimmung einzuholen, die wiederum in der Folge der Nürnberger Ärzteprozesse $1947 \mathrm{zu}$ einer entsprechenden Selbstverpflichtung der Ärzte auf internationaler Ebene ausgebaut

4) Vgl. im Bezug auf den gesundheitspolitischen Bereich Döhler / Manow 1997.

5) Ich wähle die männliche Form der sprachlichen Einfachheit halber für beide Geschlechter, sofern keine inhaltliche Differenzierung erforderlich ist. 
wurde. 1964 setzte die World Medical Association mit der Deklaration von Helsinki diese Entwicklung fort (Vgl. Boulton / Parker 2007). Dabei wurden die research subjects zu Teilnehmern und Partnern umbenannt. ${ }^{6}$

In der Chirurgie ist IC eine gängige Praxis, die jedoch den Arzt und weniger den Patienten schützt: Der Operateur lässt sich vor der Operation schriftlich bestätigen, dass er den Patienten über die Behandlungsmöglichkeiten und deren Risiken aufgeklärt hat und dass der Patient mit dem gewählten Eingriff einverstanden ist. ${ }^{7}$ So weit zum IC.

Shared decision making bezeichnet ${ }^{8}$ weitergehend ,eine Form der Arzt-Patient-Kommunikation, die zum Ziel hat, den Wunsch des Patienten nach Beteiligung an den Entscheidungen über sein Gesundheitsproblem zu realisieren. Arzt und Patient kommunizieren auf einer partnerschaftlichen Ebene über die objektiven und subjektiven Aspekte einer anstehenden Entscheidung" (Klemperer / Rosenwirth 2005: 6). ${ }^{9}$ SDM ist durch vier Charakteristika gekennzeichnet: Die Entscheidung zweier Personen, des Patienten und des Arztes, die aktive Beteiligung beider am Prozess der Entscheidungsfindung, die gegenseitige Informierung im Verlauf des Prozesses, die Zustimmung zur Behandlungsentscheidung und Bereitschaft zur aktiven Umsetzung (Vgl. Scheibler et al. 2005: 24, nach Charles et al. 1999). Als Ziele des SDM nennen Klemperer / Rosenwirth (Vgl. 2005: 6) erstens, den Wunsch der Patienten nach Beteiligung an den Entscheidungen über sein Gesundheitsproblem zu erfüllen. Zweitens soll eine hohe Entscheidungsqualität erreicht werden, ,indem aufgrund neuester, evidenzbasierter Informationen Entscheidungen getroffen und umgesetzt werden, die mit den Präferenzen der Patienten übereinstimmen. Das SDM-Konzept beinhaltet somit die Patienten- und Outcome-Orientierung sowie eine wissenschaftliche Grundlage“. ${ }^{10}$

Diese Kritik des paternalistischen Modells und die Entwicklung zum SDM fanden allerdings nicht im medizinsoziologischen Elfenbeinturm statt, sondern reagierten auf soziale Bewegungen. In den USA kann man zuerst beobachten, wie die medizinische Wissenschaft Impulse aus sozialen Bewegungen aufnahm. Bereits 1974 stellten die Soziologen Gartner und Riesman eine entstehende Selbsthilfebewegung zu medizinischen Belangen vor. Sie gaben einen Überblick über diese Bewegung in den amerikanischen Städten und sahen in ihr eine Vorhut aktiver Konsumenten. Die Impulse wurden in der medizinischen Wissenschaft aufgenommen. In den 1980er Jahren entwickelte die Medizinprofessorin Kate Lorig (Stanford University) ein Chronic Disease Self-Management Programme (Vgl. Lorig / Holman 1989; Lorig et al. 1993), das viel Beachtung fand. Ähnlich entstand auch in Deutschland eine Selbsthilfe-Bewegung, die wiederum in der Wissenschaft auf Resonanz stieß (Vgl. Badura / v. Ferber 1981; Kickbusch / Trojan 1981).

In Großbritannien wurde 1983 das College of Health, das mit der Consumers' Association verbunden war, mit dem Ziel gegründet, die Interessen der Patienten zu vertreten und sie

6) Vgl. Miller / Boulton 2007. Ein Beispiel zum IC im Verbraucherrecht: Das österreichische Bundesgesetz gegen unlauteren Wettbewerb von 1984 wurde 2007 um die Definition ergänzt, eine unlautere Geschäftspraktik beeinträchtige die Fähigkeit des Verbrauchers, eine informierte Entscheidung zu treffen.

7) Daher warnen Meisel / Kuczewski (1996) vor dem „Mythos“, IC sei lediglich ,a medical miranda warning".

8) Zur Operationalisierung vgl. Elwyn et al. 2003; Faller 2003; Scheibler 2004. Das Konzept wird inzwischen auch im Schulmanagement genutzt.

9) Vgl. als frühe Forderung Brody 1989; als Modell Emanuel / Emanuel 1992; zur Entwicklung Charles / Gafni 1999.

10) Klemperer / Rosenwirth 2005: 14, mit Berufung auf Institute of Medicine 2001. Dass mit der evidence-based medicine neue Verwaltungseliten entstehen, die die Patientenorientierung konterkarieren können (Vgl. Vogd 2002), wird nicht bedacht. 
stärker in die Belange der Gesundheitsversorgung einzubinden. In den späten 1980er Jahren erfuhr die Konsumententerminologie eine Thatcheristische Wendung: Medizinische Dienstleistungen werden dabei zu einer Ware, die - wie jede andere auch - effizient produziert und unter Wettbewerbsbedingungen eines freien Marktes konsumiert werden kann (Vgl. Logan et al. 1989). Somit treten also auch Patienten als homines oeconomici in Erscheinung und eignen sich als rationale Evaluatoren des Gesundheitssystems (Vgl. Meredith 1996). Die Idee, eine entsprechende Orientierung der Patienten voranzutreiben, fand 1999 in ein White Paper der britischen Regierung mit dem Titel Saving Lives: Our Healthier Nation Eingang. Sie wurde 2001 vom Department of Health in einer Denkschrift The Expert Patient aufgenommen. ${ }^{11}$ Dort lesen wir:

„Research and practical experience in North America and Britain are showing that today's patients with chronic diseases need not be mere recipients of care. They can become key decision-makers in the treatment process. By ensuring that knowledge of their condition is developed to a point where they are empowered to take some responsibility for its management and work in partnership with their health and social care providers, patients can be given greater control over their lives. Self-management programmes can be specifically designed to reduce the severity of symptoms and improve confidence, resourcefulness and self-efficacy." $(5)^{12}$

So hat also eine medizinische Selbsthilfebewegung in der Wissenschaft dahingehend Anschluss gefunden, dass sie als Avantgarde rationaler Konsumenten gedeutet wurde. Deren Rationalität ermöglichte die Übernahme von Eigenverantwortung. Die Rezipienten-Haltung vieler Patienten sollte mit self-management-Programmen zu einer nicht nur personalen, sondern auch medizinischen efficacy ${ }^{13}$ insbesondere chronisch Kranker verändert werden.

Um das Jahr 2000 wurden diese Impulse auch in Deutschland aufgenommen. In das die Belange der Krankenkassen regelnde Sozialgesetzbuch V wurde mit der Gesundheitsreform des Jahres 2000 eine Bestimmung eingefügt, die die Spitzenverbände der Krankenkassen verpflichtete, ,im Rahmen von Modellvorhaben [...] Einrichtungen zur Verbraucher- und Patientenberatung“ einzurichten, ,die sich die gesundheitliche Information, Beratung und Aufklärung von Versicherten zum Ziel gesetzt haben“ ( $\$ 65$ b). Eine Arbeitsgruppe der Stuttgarter Akademie für Technikfolgenabschätzung (Vgl. Dierks et al. 2001) vereinte Gesundheitswissenschaftler, Vertreter der Selbsthilfegruppen und Kommunalpolitiker unter der Programmatik „Gesundheitliche Kompetenz erhöhen, Patientensouveränität stärken“. Dieses Motto ist von vielen Gesundheitsämtern und zum Beispiel von der „Gesellschaft für Versicherungswissenschaft und -gestaltung“ (2007) aufgenommen worden. Der Evaluationsbeirat im „Forum gesundheitsziele.de“ hat 2006 das Konzept von Patientensouveränität näher zu umreißen versucht. Die Gesundheitswissenschaftler v. Reibnitz, Schnabel und Hurrelmann (2001) erörterten „Möglichkeiten und Grenzen der Patientenberatung“ unter dem Titel „Auf dem Weg zum ,souveränen“ Patienten“, der im Text durch Informiertheit, empowerment etc. charakterisiert wird. ${ }^{14}$

11) Ähnliche Gedanken verfolgt der King's Fund mit dem Programm „Promoting Patient Choice“ (Vgl. Richards 1998). In den USA propagiert die United States Preventive Services Task Force das shared medical decision making (Vgl. Kaplan 2004).

12) Entsprechend wurden self-management-Programme für Patienten mit Arthritis, manischer Depression und multipler Sklerose empfohlen.

13) Efficacy bedeutet Effizienz auf technischer, efficiency darüber hinaus auch auf ökonomischer Ebene.

14) Im gesundheits- und pflegewissenschaftlichen Kontext wird häufig von der angeblich paternalistischen Patienten- auf die angeblich Autonomie anzeigende Kunden-Semantik umgestellt. Organisationssoziologisch betrachtet geht es einer kundenorientierten ökonomischen Organisation jedoch ,um die Verwandlung bloßer Käufer, die als solche preisbewusste Entscheidungen treffen, in Kunden, die von Preisvergleichen absehen und sich stattdessen an die Organisation binden." (Tacke / Wagner 2005: 134). Ähnlich schildert Vogd (2004: 154) die Patienten- und die Kundenrolle als komplementär. 
Seither sind viele weitere Bestrebungen zur Implementation des SDM auf verbandlicher und politischer Ebene zu beobachten, die miteinander in einem korporativen Geflecht verbunden sind. Seit 2003 sind Patientenverbände im „Gemeinsamen Bundesausschuss“ $(\mathrm{GBA})^{15}$ vertreten. Dieser gesundheitspolitische Spitzen-Ausschuss ist eine eigenständige juristische Person des öffentlichen Rechts unter der Rechtsaufsicht des Bundesministeriums für Gesundheit (BMG). Zu seinen Hauptaufgaben gehört der Erlass von Richtlinien vor allem zur Qualitätssicherung in verschiedenen Sektoren des Gesundheitswesens. Die von ihm beschlossenen Richtlinien sind für alle Akteure der Gesetzlichen Krankenversicherung (GKV) bindend. Der GBA besteht aus 21 Mitgliedern:

- Neun Vertretern der Spitzenverbände der gesetzliche Krankenkassen,

- Neun Vertretern der ärztlichen Spitzenverbände und

- Drei „unparteiischen“ Mitgliedern, häufig Wissenschaftlern.

Dazu kommen Patientenvertreter, die mit Antrags- und Beratungs-, nicht jedoch mit Stimmrecht ausgestattet sind. Gegenwärtig werden sie von folgenden Dachverbänden benannt:

- Vom Deutschen Behindertenrat (DBR),

- Von der BundesArbeitsGemeinschaft der PatientInnenstellen (BAGP),

- der Deutschen Arbeitsgemeinschaft Selbsthilfegruppen e. V. und

- der Verbraucherzentrale Bundesverband e. V.

Diese Organisationen, so heißt es auf der entsprechenden Website, ,bilden mit den in ihnen vertretenen Mitgliedern die Vielschichtigkeit der Patientenorganisationen und der Selbsthilfe $a b$ “. Eine solche Aussage ist charakteristisch für korporatistische Politik, in der hoch aggregierte Verbände mit staatlichen Organisationen kooperieren. Dieses Politikmodell, das klassisch mit sozialdemokratischer Politikgestaltung verbunden war, wurde häufig als immobil und die Betroffenen mediatisierend, jedenfalls als in der Krise befindlich bezeichnet (Vgl. Streeck 2005; Weßels 2000). ${ }^{16}$ In der Gesundheitspolitik scheint es jedoch auch nach dem Ende der sozialdemokratisch geführten Bundesregierung im Jahre 2005 dominant. 2004 wurde das Amt einer Beauftragten der Bundesregierung für die Belange der Patientinnen und Patienten eingeführt. ${ }^{17}$ Sie sieht ihre Aufgabe u. a. darin, für die Patienten und Patienten mehr Transparenz und Qualität(ssicherung) im Gesundheitswesen zu erreichen.

Die Richtlinien-Politik des „Gemeinsamen Bundesausschusses“ wird im „Programm für Nationale VersorgungsLeitlinien “ fortgeführt, das die ärztlichen Spitzenverbände tragen und an dem sich die Selbsthilfedachverbände beteiligen. In diesem Programm geht es speziell um die Erarbeitung von „PatientenLeitlinien“, d.h. um die Bereitstellung einer „Entscheidungshilfe über die angemessene ärztliche Vorgehensweise bei speziellen gesundheitlichen Problemen", die auf evidenzbasierter Medizin beruht. ${ }^{18}$

Auch auf nationalstaatlicher Ebene findet Patientenbeteiligung statt. So haben im Jahre 2006 die Bundesministerien für Gesundheit (BMG), Bildung und Forschung und Arbeit und Soziales gemeinsam ein sechsjähriges Forschungsprogramm ausgeschrieben, das u.a. die „Organisation einer partizipativen Versorgungsgestaltung“ vorantreiben soll. ${ }^{19}$ Das BMG hat einen eigenen Förderschwerpunkt „Patient als Partner im medizinischen Entscheidungsprozess“ eingerichtet. ${ }^{20}$

15) Der GBA ist Nachfolger der „Konzertierten Aktion im Gesundheitswesen“, deren korporatistischer Charakter bereits im Namen aufscheint (Vgl. GBA).

16) Jessop (1990: 124ff) schrieb bereits 1990 über Grenzen des Korporatismus und deutete ihn später als workfare-Strategie, d.h. als Übergang zu einer rigiden Form von Sozialpolitik.

17) Das Amt hat seit 2004 die SPD-Politikerin Dipl.-Psych. Helga Kühn-Mengel inne.

18) Vgl. Methoden-Report Patientenbeteiligung (2007: 7).

19) Vgl. Patient als Partner.

20) Siehe Fußnote 9; Im Mai 2007 fand die 5. Tagung dieses Förderschwerpunktes in Freiburg statt. 
Entwickelte die Selbsthilfebewegung der 1980er Jahre eine bottom-up-Mentalität, in der Misstrauen gegenüber möglichen Abhängigkeiten von „Staatsknete“ herrschte und die Kooperation mit professionellen Organisationen abgelehnt wurde, so finden wir in diesen neueren korporatistischen Entwicklungen eine top-down-Gesundheitspolitik, die weniger auf vorhandene Partizipationsfähigkeit medizinischer Laien als auf deren empowerment sowie auf advocacy für weniger Partizipationsfähige setzt. Das legt die Frage nahe, wie sich das Verhältnis von Laien- und Expertenwissen zunächst unabhängig von dieser advocacy gestaltet.

\section{Nähert sich in medizinsoziologischer Wahrnehmung das Laien- dem Experten- wissen?}

Das Verhältnis von (medizinischen) Experten und Laien ist ein strittiges Thema der Medizin- und der Wissenssoziologie (Vgl. Stollberg 2001: 55ff). Die einen Autoren arbeiten die Unterschiede heraus, die anderen die Ähnlichkeiten. Hahn et al. (1999: 77) betonen, dass der Patient seiner Krankheit einen Sinn geben wolle, während den Ärzten ihre medizinisch-wissenschaftliche Berufsorientierung derlei Spekulationen verbiete. Sie hätten die Krankheit zu diagnostizieren und für Linderung und Abhilfe zu sorgen. Diese These ist jedoch problematisch. Erstens unternehmen auch Ärzte Sinnkonstruktionen, die von denen der Patienten in der Sachdimension getrennt sind (Vgl. allgemein Luhmann 1984: 92ff): Den Ärzten geht es z.B. um Symptome, die für sie auf die jeweilige Krankheit verweisen; den Patienten geht es z.B. um die Bedeutung der Krankheit für ihren Lifestyle oder auch für den Sinn ihres Lebens. Zweitens gehört zur Arztrolle der Umgang mit Sinnkonstruktion der Patienten. Denn die Expertenrolle des Arztes als eines Professionellen ist von der des (medizinischen) Wissenschaftlers zu unterscheiden. Empirisch gibt es innerhalb des Systems der Krankenversorgung durchaus eine Bearbeitung der Sinnkonstruktionen der Patienten, die häufig vom Pflegepersonal vorgenommen wird (Vgl. z.B. Strauss / Fagerhaugh / Suczek / Wiener 1980). Auch zeigt Lutfey (2005), dass das medizinische Personal in Diabetes-Kliniken die Rollen von Erziehern, Detektiven, Kaufleuten, Polizisten und cheerleaders übernimmt.

Andere Autoren heben hervor, dass keine klare Wissensteilung zwischen Patienten und Ärzten bestehe. Willems (1992) spricht von einer fraktalen Wissensverteilung zwischen Patient, Allgemein- und Facharzt. ${ }^{21}$ Williams und Calnan (1996) sehen ein re-skilling der Patienten in der Gegenwart. Tuckett et al. (1985) bezeichnen gar Arztkonsultationen als „,meetings between experts“. Gülich (1999: 187) überlegt, ,daß für die Kommunikation vor allem wichtig ist, wie Experten- und Nicht-Expertenrollen in der Interaktion konstituiert werden“". Demnach wäre also zu erwarten, dass kommunikativ aktives Patientenverhalten vom Arzt eliziert wird. Jedoch haben empirische Studien verwickelte Verhältnisse ergeben. Zu mehr als $90 \%$ erteilten Ärzte von sich aus und ohne auf entsprechende Nachfragen von Patientenseite Informationen; zu mehr als $90 \%$ partizipierten Patienten von sich aus aktiv an den Gesprächen (Vgl. Gordon / Street / Kelly / Souchek / Wray 2005: 1022). Ärzte, die eher partnerschaftlich orientiert waren, verhielten sich in Arzt-Patient-Gesprächen nicht signifikant unterschiedlich zu Ärzten, die an professioneller Kontrolle festhalten wollten (Vgl. Street / Krupat / Bell / Kravitz / Haidet 2003: 613f).

Eine besondere Rolle bei der potenziellen Expertisierung der Patienten spielt die Nutzung des Internets. ${ }^{22}$ Die bildungs- und geschlechtsspezifischen biases dieses Mediums dürften

21) Willems (1992: 113) analysierte die Kommunikationen zwischen einem Facharzt, einem Allgemeinmediziner und ihrer Patientin mit dem Resultat: ,What happens (...) is a modification of the division of knowledge and skills between physicians and patients. A clear division between their seemingly fundamentally different competences becomes more and more fractal“. Auch Giddens spricht von einer arbeitsteiligen Organisation des Expertentums (1996: 164).

22) Bei Coulter und Magee (2003) wird das Internet als nur marginale Informationsquelle genannt. Vgl. Abschnitt 3. 
mit der Zeit geringer werden. Fox et al. (2005) untersuchten ein interaktives Pharma-Diskussions-Forum. Sie fanden dort zwar viele Patienten-Experten. Der Diskurs war aber auf die Pharma-Nutzung und eine somatische Perspektive verengt. Nettleton und Hanlon (2006: 70) fassen eine Reihe von Studien dahingehend zusammen, dass Patienten das Internet nutzten, um Symptome zu klären und zu bewerten. Die Patienten wollten „gute“ Patienten werden, stellten die Internet-Dienste des britischen Nationalen Gesundheitsdienstes NHS in dessen allgemeinen Kontext und vertrauten den medizinischen Experten weiterhin.

Es lässt sich gegenwärtig nur eine vorläufige Schlussfolgerung aus der ExpertisierungsDebatte ziehen. Vielleicht sind viele Ärzte weniger autoritär, als vielfach erwartet wird. Ähnlich sind auf der anderen Seite viele Patienten eigenständig informiert, erwarten jedoch eine Bewertung dieser Informationen durch den Arzt.

\section{Empirische Studien zu Patientenbedürfnissen hinsichtlich Information und Beteiligung}

Die Patientenseite will ich nun näher beleuchten. Zunächst sei eine international vergleichende Studie „The European Patient of the Future" referiert, in deren Rahmen im Jahre 2002 jeweils 1000 Patienten in acht Ländern befragt wurden. ${ }^{23}$ Die Autoren gingen von der These aus, die Gesundheitsdienste aller europäischen Länder erführen einen deutlichen Nachfrageschub, der zum einen aus der demographischen Entwicklung (Zunahme Älterer an der Gesamtbevölkerung) und zum anderen aus einem gestiegenen Bewusstsein dafür resultiere, was auf dem medizinischen Markt angeboten werde. „Consumerism is apparent in many areas of public life and health care is no exception" (Coulter / Magee 2003: 1). Was nun die Resultate der Studie anbelangt, waren nahezu alle deutschen Interviewten mit ,ihrem“ Arzt zufrieden, auch wenn sie bisweilen über Zeitmangel und wenig individuelle Kommunikation in den Arztkontakten klagten (Vgl. ebd.: 37). Diese Zufriedenheit äußerte sich z.B. hinsichtlich medizinischer Informationen darin, dass $58 \%$ angaben, den Informationen ihres Haus- respektive Facharztes zu vertrauen. Bücher rangierten mit $17 \%$ deutlich hinter den Ärzten; das Internet tauchte als Informationsquelle und Vertrauensgegenstand nur bei Jüngeren auf (Vgl. ebd.: 43). ${ }^{24}$

In den acht Vergleichsländern sagten insgesamt nur $36 \%$ der Befragten, die Ärzte hörten sorgfältig zu, gäben die Möglichkeit, Fragen zu stellen, und erklärten die Sachlage ausführlich (Vgl. ebd.: 208). ${ }^{25}$ Dennoch wurden als Hauptinformationsquelle für medizinische Problemlagen und Therapiemöglichkeiten auch im internationalen Vergleich mit $84 \%$ die Ärzte genannt, weit danach Zeitungen und Zeitschriften sowie mit $10 \%$ das Internet (Vgl. ebd.: 212). Was therapeutische Entscheidungen betrifft, sahen $23 \%$ sich selbst und $26 \%$ die Ärzte als diejenige Person, die die Entscheidungen fällen sollte, während sich $51 \%$ für eine gemeinsame Entscheidung von Arzt und Patient aussprachen. Ältere Patienten bevorzugten eine allein ärztliche Entscheidung, gebildetere eine gemeinsame von Arzt und Patient (Vgl. ebd.: 218f).

Flynn et al. konstruierten vier Typen partizipativer Patienten: „non-deliberative delegators“ (23\% der Befragten) ,preferred low deliberation and doctor control over decisions while deliberative delegators“ (16\% der Befragten) ,preferred high deliberation and doctor control. Non-deliberative autonomists“ (11\% der Befragten) „,preferred low deliberation and personal control over decisions while deliberative autonomists“ (46\% der Befragten) ,pre-

23) Die Länder waren Deutschland, Italien, Polen, Slowenien, Spanien, Schweden, Schweiz und das Vereinigte Königreich. Auf nationale Differenzen werde ich nicht speziell eingehen.

24) Auch Sillence, Briggs, Harris und Fishwick (2007) fanden in ihrer Studie über Internet-Nutzung von Patienten den Arzt als deren primäre Informationsquelle.

25) Die positiven Antworten waren im Vereinigten Königreich, der Schweiz, Italien und Spanien deutlich häufiger als in Polen und Deutschland. Jedoch geht es hier nicht um mögliche - wie immer begründete - nationale Unterschiede. 
ferred high deliberation and personal control“" (Flynn et al. 2006: 1163). Wir haben bereits bei Coulter und Magee (2003) gesehen, dass die jeweils größte Gruppe der Befragten, wenn nach Beteiligungswünschen gefragt wird, angibt, das SDM-Modell zu präferieren.

Dennoch scheint die Schlussfolgerung von Loh, Simon, Kriston und Härter (2007: A 1483) problematisch, ,dass Patienten mehrheitlich bei Behandlungsentscheidungen im ärztlichen Gespräch explizit beteiligt werden wollen“. Vielmehr zeigen Coulter und Magee (2003) zwar, dass Klagen über das Kommunikationsverhalten der Ärzte verbreitet sind, diese aber dem Vertrauen in die medizinische Sach- und die Entscheidungskompetenz keinen Abbruch tun. Von den Ärzten unabhängige Informationsquellen werden vergleichsweise selten genutzt. Obgleich nur $26 \%$ der befragten Patienten der Ansicht waren, dass der Arzt die wesentlichen therapeutischen Entscheidungen fällen sollte, blieb der kritische Impetus dieser Meinung aus, denn die Hälfte der Befürworter sah SDM als in ihrem Verhältnis zu ihren Ärzten realisiert an.

Empirische Studien medizinischer wie soziologischer Provenienz berichten von einem deutlichen Vertrauensverhältnis der Patienten zu ihren Ärzten. So besagt eine niederländische Studie, ${ }^{26}$ dass eine Patient-Arzt-Beziehung, die auf gegenseitigem Vertrauen und Respekt beruht, mehr Rückhalt bei den Befragten fand als das kritische Konsumenten-Verhalten, von dem Coulter und Magee hypothetisch ausgingen. In Deutschland ist der Arztberuf seit mindestens vier Jahrzehnten unangefochten der am meisten angesehene Beruf. ${ }^{27}$ Daher ist es kein Wunder, dass diesem Stande ${ }^{28}$ auch Vertrauen entgegengebracht wird: Ihrem Hausarzt vertrauen $90 \%$ der Patienten, den Ärzten allgemein $80 \%{ }^{29}$

26) Bei de Ridder, Depla, Severens und Malsch (1997: 557) findet sich die folgende Aufstellung über priorities in belief on coping with the health care system $(n=87)$ (es geht um Präferenzen; am letzten Platz die am wenigsten ideale Attitüde):

$A b b$ 1: priorities in belief on copiing with the health care system

\begin{tabular}{|l|l|}
\hline $\begin{array}{l}\text { Professional } \\
\text { consultation }\end{array}$ & 4.0 \\
\hline Engagement & 3.9 \\
\hline Seek advice & 3.8 \\
\hline Trust & 3.3 \\
\hline Demand & 3.2 \\
\hline Self-assertion & 3.2 \\
\hline Commitment & 3.0 \\
\hline $\begin{array}{l}\text { Seek } \\
\text { alternatives }\end{array}$ & 2.3 \\
\hline Seek support & 2.2 \\
\hline Powerlessness & 1.9 \\
\hline
\end{tabular}

27) Im Jahre 2005 mit $71 \%$ vor dem der Krankenschwester mit 45, des Polizisten mit 40 und des Hochschulprofessors mit $36 \%$ : Allensbacher Berichte Nr. 12 / 2005.

28) Im Deutschen werden Ärzte gern als Stand bezeichnet. Damit ist offenbar nicht Status, sondern die Begrifflichkeit Max Webers gemeint. D.h. es wird von einer positiven ständischen Sonderschätzung der Ärzte ausgegangen. Dies illustriert die Übersetzung des Buchtitels von Freidson: Aus Profession of medicine wurde Der Ärztestand.

29) So die Institute Medvantis und INRA 2001 laut www.verbrauchernews.de/artikel/000000919.html, abgerufen am 29.9.05. Ähnliche Zahlen bei Kröhn, Tolksdorff, Matyslak-Klose, Heintze, Wolf, Kensche, Howorka, Wehrmeyer, Braun (2004). Jedoch wünschten mehr als $60 \%$ mehr Aufklärung durch ihren Arzt, und $20 \%$ hatten kein oder nur geringes Vertrauen in ihre Ärzte (Vgl. Richter-Hebel 1999). 
Medizinsoziologische Studien zeigen durchwegs eine sehr niedrige Patientenbeteiligung an medizinischen Entscheidungen, die sie betreffen (zum Überblick Collins / Drew / Watt / Entwistle 2005: 2611). Nur 37 \% deutscher Patienten informierten sich vor dem Praxisbesuch über die Praxis oder über ihre Erkrankung, und diese holten ihre Informationen zu $50 \%$ von Ärzten und nur zu $33 \%$ von anderen Patienten. Das Hauptinteresse der Informationsbeschaffung galt mit $62 \%$ der Arztwahl, dagegen mit nur $21 \%$ der eigenen Erkrankung. Nur knapp $3 \%$ investierten mehr als drei Stunden in die Informationsbeschaffung (Vgl. MojonAzzi / Wagner / Mojon 2003). $95 \%$ wünschten in der ärztlichen Praxis, davon $75 \%$ vom Arzt selbst informiert zu werden. Laut Bertelsmann Gesundheitsmonitor stehen $41 \%$ der befragten Patienten, die sich über Behandlungsmaßnahmen informierten, $32 \%$ gegenüber, die keinerlei Informationen gesucht hatten (Vgl. Klemperer / Rosenwirth 2005: 23). Eine Studie aus St. Gallen über ophthalmologische Patienten formuliert zugespitzt:

„Die meisten (...) Patienten entsprechen nicht dem Bild des informierten und mündigen Patienten und sind dementsprechend ohne zusätzlichen Wissenserwerb nur mit Unterstützung von Gesundheitsfachpersonen in der Lage, die Verantwortung für die eigene Gesundheit im Sinne des Empowerments zu übernehmen“ (Mojon-Azzi / Wagner / Mojon 2003: 487).

Also scheint konsumistisches Verhalten wenig verbreitet: Die Patienten entwickeln nicht nur Vertrauen in die Sachkompetenz der Ärzte, sie nutzen diese auch als zentrale Informationsquelle in medizinischen Dingen und überlassen ihnen medizinische Entscheidungen. Marstedt (2003) kommt in seinem Überblick über die deutschsprachige Forschungsliteratur zu dem Resultat, dass SDM zwar heute noch kein Standard in der ärztlichen Sprechstunde sei, wohl aber der ,informierte Patient“. Deber, Kraetschmer und Irvine (1996) unterscheiden in ihrer Untersuchung von SDM zwischen Informationsbedürfnis und Entscheidungsteilhabe der Patienten. Sie fanden ein hohes Informations- bei niedrigem Teilhabebedürfnis (Vgl. auch O'Donnell / Monz / Hunskaar 2007).

Bei einer Gruppe von Patienten ließen sich in besonderer Weise informierte oder gemeinsame Entscheidungsfindungsprozesse erwarten: Bei den Patienten nicht-schulmedizinischer ${ }^{30}$ Medizinformen. Denn diese Patienten haben sich nicht nur den Arzt, sondern auch die Heilmethode ihrer Wahl entschieden und müssen diese häufig zumindest teilweise selbst bezahlen. Robert Frank und ich haben Patienten indischer und chinesischer Medizin in Deutschland befragt (Vgl. Frank / Stollberg 2004) und sind dabei keineswegs überwiegend auf ,aktive Konsumenten“31 gestoßen. Vielmehr scheint zu ihrer Beschreibung das paternalistische Modell am ehesten geeignet zu sein, da die Kontrolle über therapeutische Alternativen beim Arzt verbleibt. Aus den Berichten der Patienten wird deutlich, dass sie ihre Bedürfnisse als wahrgenommen und ihre Perspektiven als einbezogen wahrnehmen. Somit erscheinen Konsultationen in asiatischer Medizin als paradox: paternalistisch und patientenzentriert. Hierfür als Beispiel eine Ayurveda-Patientin:

„My gastro-enterologist didn't give me a helping hand when it came to nutrition, which would have been very helpful to me. He only said: ,We have to try several drugs and see which one you tolerate. 'And in the introductory conversation with my Ayurvedic physician, it became evident that Ayurveda offers much more in that respect, it doesn't just focus on medical aspects, pharmaceutical chemistry, prescribing products, but it always involves gi-

30) Die üblichen Bezeichnungen ,alternative“ oder „,komplementäre“ Medizin zielen auf die Anwendungsarten in Relation zur „Schul“medizin. Ich präferiere daher den Begriff „,heterodoxe Medizin“.

31) Kelner und Wellman (1997) nennen die von ihnen interviewten kanadischen Patienten heterodoxer Medizin, die die Behandlung aus eigener Tasche bezahlen müssen, ,smart consumers“. Giddens (1997: 138f) betont die aktive Grundhaltung dieser Patienten. 
ving advice, which doesn't cost anything and which fits into my daily life. And that is the big difference to other physicians who prescribe any old drug and leave you out in the cold." (Frank / Stollberg 2002: 236).

Die empirischen Studien lassen sich dahingehend resümieren, dass die meisten Patienten ein hohes Informations-, jedoch ein geringeres Teilhabebedürfnis an Entscheidungen ausprägen.

\section{Schlussfolgerungen zum Vertrauensbegriff und zu SDM als modernem Mythos der Medizin}

Diese Schlussfolgerung aus empirischen medizinsoziologischen wie medizinischen Studien will ich nun in theoretische Erörterungen zum Vertrauen in der „Gesundheitsgesellschaft“ einbetten.

Giddens hat die Weiterentwicklung des Arzt-Patient-Verhältnisses im Zuge der Enttraditionalisierung der Gesellschaft erörtert. Bei der Herausbildung post-traditionaler sozialer Beziehungen spiele aktives Vertrauen eine große Rolle.

„In manchen Zusammenhängen bleibt uns heute keine andere Entscheidung, als uns zu entscheiden und uns dabei auf Expertenwissen zu stützen, das wir ganz unterschiedlichen Quellen entnommen haben“ (Giddens 1996: 321).

Zwar stellt auch Giddens in der argumentativen Tradition des Systemvertrauens die ,institutionalisierte Reflexivität“" ins Zentrum seiner Argumentation; als Nebenprodukt spricht er aber über eine Veränderung des Vertrauens in der Folge der Pluralisierung von Expertenmeinungen. ${ }^{32}$ Er nennt dieses veränderte Vertrauen ,aktiv“. Globalisierung und Enttraditionalisierung förderten einerseits das Eindringen der Wissenssysteme von Experten in viele Aspekte des modernen Alltagslebens. Diese Invasion verschränke sich jedoch andererseits mit Reflexivität, und insbesondere für das Gebiet der Medizin sei eine Skepsis der Laien bezeichnend, die Vertrauen in den Arzt als Experten nicht unterhöhle, sondern vielmehr in eine aktive Haltung verwandele:

„Alle Formen von Expertentum setzen aktives Vertrauen voraus, denn hier tritt jeder Anspruch auf Autorität neben den weiterer Autoritäten, und oft sind die Experten selbst unterschiedlicher Meinung (...). Das Ansehen der Wissenschaft selbst, das in früheren Stadien der Entwicklung der modernen Institutionen von entscheidender Bedeutung war, wird von eben der skeptischen Einstellung untergraben, die selbst der Antrieb des wissenschaftlichen Vorgehens ist. [...] Wer z.B. Probleme mit seiner Gesundheit hat, hält sich auch heute noch vielleicht zunächst an die Schulmedizin und deren Technologie, um diese Probleme loszuwerden. [...] Vor dem Hintergrund aktiver Vertrauensmechanismen könnte es aber auch sein, daß sich der Betreffende entscheidet, eine zweite oder dritte Diagnose einzuholen." (Giddens 1997: 138f)

Diesen Umgang mit der Pluralität von Experten und Expertenmeinungen nennt Giddens aktives Vertrauen. Mir scheint es jedoch günstiger, die Unterscheidung von aktiv und passiv auf Typen von Verhaltensweisen zu beziehen, ${ }^{33}$ wie dies mit dem Begriff des aktiven Konsumenten geschieht. Dann charakterisiert aktives Vertrauen nicht die Patienten in einer ent-

32) Zwar erwähnt auch Luhmann (1973: 52) die funktionale Spezifikation des Vertrauens ,,auf Kommunikationen bestimmter Art, für die der andere nachweisbar kompetent ist“". Er reflektiert jedoch in diesem Zusammenhang nicht die Divergenz von Expertenmeinungen.

33) Vgl. die Antwort von Salentin (2004: 114) auf die Frage, ob sich Migranten in ,ethnische Kolonien' zurückziehen: „Vielmehr sind die gut in die Migrantengesellschaften Integrierten tendenziell auch eng mit der Mehrheitsgesellschaft verbunden.“ 
traditionalisierten Gesellschaft insgesamt, sondern es gibt aktive und passive Patienten. Die treuen und compliance übenden gehören zu den passiven, die smart consumers dagegen $\mathrm{zu}$ aktiven, SDM wünschenden Patienten.

SDM kann zusammengefasst als „moderner Mythos der Medizin“ begriffen werden, als ein praktikables Schema, das ein Versprechen formuliert (Vgl. Vogd / Saake 2008: 22). Die Unsicherheit einer Krankheitssituation kann nicht unmittelbar durch medizinische Definition und Intervention, sondern soll durch Rekurs auf Partizipation entschärft werden. Diese Fassung des Mythos-Begriffs geht von der neo-institutionalistischen Perspektive aus: Nach Meyer und Rowan (1977: 343ff) identifizieren moderne Mythen soziale als technische Zwecke. Die adäquaten Mittel, diese technischen Zwecke zu erreichen, werden als rationale spezifiziert. Sie sind stark institutionalisiert und bedürfen keiner weiteren Legitimation. Professionen seien z.B. eine soziale Struktur, deren Institutionalisierung durch Ausbildung und Zertifizierung oft wichtiger genommen werde als ihre Effektivität. ${ }^{34}$

Stärker als Meyer und Rowan akzentuiere ich jedoch auf das Systemvertrauen, das sich in dieser Anerkennung der institutionalisierten Expertenschaft ausdrückt. Ich habe nach den gegenwärtigen Ausprägungen dieses Systemvertrauens gefragt und nenne nicht die Profession einen Mythos, sondern die Relativierung ihrer Bedeutung durch partizipative Formen wie IC und SDM. Sie versprechen zwar nicht unmittelbar verbesserte Heilung durch Partizipation, ${ }^{35}$ dehnen aber die im politischen System heimischen partizipatorischen Erwartungen auf die Medizin aus. Dies entspricht den Patientenwünschen, wie sie mehrheitlich auf die Frage geäußert werden, wer die therapeutischen Entscheidungen fällen solle. Zwar wünschen die meisten Patienten Informationen über Diagnose und Behandlungsmöglichkeiten der jeweiligen Krankheit und beschaffen sie sich mehr oder minder häufig. Sie wünschen aber auch eine Bewertung dieser Informationen durch den Arzt, dem sie hinsichtlich der Auswahl und Durchführung der Therapie letztlich vertrauen. Dies scheint mir übrigens durchaus different zu Nichtwählern im politischen System zu sein: Es gibt keine Ablehnung oder Verachtung einer „medizinischen Klasse“. 36

Ich habe eingangs Kickbusch (2006: 69) dahingehend zitiert, die Gesundheitsgesellschaft bedürfe eines aktiven und kritischen Bürgers, Konsumenten und Patienten, dessen Gesundheitskompetenz gesteigert werden müsse. Mit ihren Thesen hat die langjährige Präsidentin des für Europa zuständigen Büros der Weltgesundheitsorganisation offenbar sogar die EUKommission dazu bewegen können, Pflöcke in ein gesundheitspolitisches Terrain zu schlagen, das grundsätzlich den einzelnen Mitgliedsstaaten der EU vorbehalten ist. ${ }^{37}$ Ich halte es dagegen eher mit Lupton (1997: 380), die die Entwicklung von empowerment-Politiken als Dilemma umrissen hat:

„In a sociocultural context in which autonomy and rationality are highly privileged and dependency upon others is largely viewed as evidence of weakness and irrationality, lay people may feel a continual tension between wanting to behave in a consumerist manner and avoid dependency on doctors [...], and their equally strongly felt desire [...] to take on the 'passive patient' role and invest their trust and faith in these professionals. “ 38

34) Vgl. Freidson (2001), der Professionalismus als dritten Bereich zwischen Ökonomie und Bürokratie stellt.

35) Wie hatte Luhmann (1990: 186) geschrieben: „Die politische Partei kann nicht heilen“.

36) Ansätze dazu könnte man in der öffentlichen Kritik an unterschiedlichen Wartezeiten für Angehörige gesetzlicher und privater deutscher Krankenversicherungen sehen, die auf breite Resonanz stößt.

37) Vgl. Commission of the European Communities (2007).

38) In diesen Sätzen wird deutlich, dass Selbstbestimmung ein zeitstabiles stereotypes Deutungsmuster ist (Vgl. Krähnke 2007: 185). Es passt gut zu einem Mythos der Moderne wie dem des SDM. 


\section{Literatur}

Badura, B. / C. von Ferber (1981): Selbsthilfe und Selbstorganisation im Gesundheitswesen, München.

Boulton, M. / M. Parker (2007): Informed consent in a changing environment, in: Social science and medicine 65, S. 2187-2198.

Brody, H. (1989): Transparency. Informed consent in primary care. In: Hastings Center Report 9, S. 5-9.

Charles, C. / A. Gafni / T. Whelan (1999): Decision-making in the Physician-Patient Encounter: Revisiting the Shared Treatment Decision-making Model, in: Social science and medicine 49, S. 651-661.

Collins, S. / P. Drew / I. Watt / V. Entwistle (2005): 'Unilateral' and 'Bilateral' Practitioner Approaches in Decision-making About Treatment, in: Social science and medicine 61, S. 2611-2627.

Commission of the European Communities (2007): White paper: Together for health: A strategic approach for the EU 2008-2013, abgerufen am 11.11.2008 http://ec.europa.eu/ health/ph_overview/Documents/strategy_wp_en.pdf.

Coulter, A. / H. Magee (Hrsg.) (2003): The European Patient of the Future, Berkshire.

Deber, R.B. / N. Kraetschmer / J. Irvine (1996): What role do patients wish to play in treatment decision making?, in: Archive of internal medicine 156, S. 1414-1420.

Dierks, M.-L. / E.-M. Bitzer / M. Lerch / S. Martin / S. Röseler / A. Schienkiewitz / S. Siebeneick / F.W. Schwartz (2001): Patientensouveränität. Der Autonomie Patient im Mittelpunkt, Arbeitsbericht Nr. 195 der Akademie für Technikfolgenabschätzung in Baden-Württemberg, Stuttgart.

Döhler, M. / Ph. Manow (1997): Strukturbildung von Politikfeldern: das Beispiel bundesdeutscher Gesundheitspolitik seit den fünfziger Jahren, Opladen.

Elwyn, G. / A. Edwards / M. Wensing / K. Hood / C. Atwell / R. Grol (2003): Shared decision making: Developing the OPTION scale for measuring patient involvement, in: Quality and safety in health care 12, S. 93-99.

Emanuel, E.J. / L.C. Emanuel (1992): Four models of the physician-patient relationship, in: Journal of the American medical association 267, S. 2221-2226.

Faller, H. (2003): Shared Decision Making: Ein Ansatz zur Stärkung der Partizipation des Patienten in der Rehabilitation, in: Rehabilitation 42, S. 129-135.

Flynn, K.E. / M.A. Smith / A. Maureen / D. Vanness (2006): A typology of preferences for participation in healthcare decision, in: Social science and medicine 63, S. 1158-1169.

Fox, N.J. / K.J. Ward / A.J. O'Rourke (2005): The 'expert patient': empowerment or medical dominance? The case of weight loss, pharmaceutical drugs and the Internet, in: Social science and medicine 60, S.1299-1309.

Frank, R. / G. Stollberg (2002): Ayurveda patients in Germany, in: Anthropology and medicine 9, S. 223 244.

Frank, R. / G. Stollberg (2004): Conceptualising Hybridisation - On the Diffusion of Asian Medical Knowledge to Germany, in: International Sociology 19, S. 71-88.

Freidson, Eliot (2001): Professionalism. The third logic, Cambridge.

Gartner, A. / F. Riesman (1978): Der aktive Konsument in der Dienstleistungsgesellschaft. Zur politischen Ökonomie des tertiären Sektors, Frankfurt / Main (amerikan. 1974).

Gemeinsamer Bundesausschuss (GBA), abgerufen am 27.05.2007.http://www.g-ba.de/institution/struktur/patientenbeteiligung/.

Giddens, A. (1996): Risiko, Vertrauen und Reflexivität, in: U. Beck / A. Giddens / S. Lash, Reflexive Modernisierung. Eine Kontroverse, Frankfurt / Main, S. 316-337.

Giddens, A. (1997): Jenseits von Links und Rechts, Frankfurt / Main (engl. 1994).

Gordon, H.S. / R.J. Street Jr. / P.A. Kelly / J. Souchek / N.P. Wray (2005): Physician-patient communication following invasive procedures: an analysis of post-angiogram consultations, in: Social science and medicine 61, S. 1015-1025.

Gülich, E. (1999): ,Experten’ und ,Laien': Der Umgang mit Kompetenzunterschieden am Beispiel medizinischer Kommunikation, in: Konferenz der deutschen Akademien der Wissenschaften und der Säch- 
sischen Akademie der Wissenschaften (Hrsg.), Werkzeug Sprache. Sprachpolitik, Sprachfähigkeit, Sprache und Macht, Hildesheim, S. 165-196.

Hahn, A. / W.H. Eirmbter / R. Jacob (1999): „Expertenwissen und Laienwissen. Über Deutungsunterschiede bei Krankheitsvorstellungen“, in: Jürgen Gerhards / Ronald Hitzler (Hrsg.), Eigenwilligkeit und Rationalität sozialer Prozesse. Festschrift zum 65. Geburtstag von Friedhelm Neidhardt, Opladen, S. 68-96.

Institute of medicine committee on quality of health care in America (2001): Crossing the quality chasm: A new health system for the 21 st century, Washington, DC.

Jessop, B. (1990): State theory. Putting the capitalist state in its place, Oxford.

Kaplan, R.M. (2004): Shared medical decision making. A new tool for preventive medicine, in: American Journal for preventive medicine 26, S. 81-83.

Kelner, M. / B. Wellman (1997): Health care and consumer choice: Medical and alternative therapies, in: Social science and medicine 45, S. 203-212.

Kickbusch, I. / Trojan, A. (Hrsg.) (1981): Gemeinsam sind wir stärker, Frankfurt / Main.

Klemperer, D. / Rosenwirth (2005): Shared Decision Making - ein Thema für die Selbsthilfe?, in: Deutsche Arbeitsgemeinschaft Selbsthilfegruppen (Hrsg.), Selbsthilfegruppenjahrbuch, Gießen, S. 132136.

Krähnke, U. (2007): Selbstbestimmung. Zur gesellschaftlichen Konstruktion einer normativen Leitidee, Weilerswist.

Kröhn, T. / Tolksdorff, E. / Matyslak-Klose, D. / Heintze, C. / Wolf, U. / Kensche, M. / Howorka, A. / Wehrmeyer, H. / Braun, V. (2004): Zufriedenheit von Patienten mit chronischen Darmerkrankungen in der Allgemeinmedizin, in: Zeitschrift für Allgemeinmedizin 80, S. 26-32.

Logan, J. / D. Green / A. Woodfield (1989): Healthy Competition, Sydney: Centre for Independent Societies.

Loh, A. / D. Simon / L. Kriston / M. Härter (2007): Patientenbeteiligung bei medizinischen Entscheidungen. Effekte der Partizipativen Entscheidungsfindung aus systematischen Reviews, in: Deutsches Ärzteblatt 104, A 1483- A 1489.

Lorig, K. / H.R. Holman (1989): Long-term outcomes of an arthritis self-management study; effects of reinforcement efforts, in: Social science and medicine 29, S. 221-224.

Lorig, K.R. / P.D. Mazonson / H.R. Holman (1993): Evidence suggesting that health education for selfmanagement in patients with chronic arthritis has sustained health benefits while reducing health care costs, in: Arthritis and rheumatism 36, S. 439-446.

Luhmann, N. (1973): Vertrauen. Ein Mechanismus der Reduktion sozialer Komplexität, 2. Auflage, Stuttgart.

Luhmann, N. (1984): Soziale Systeme. Grundriß einer allgemeinen Theorie. Frankfurt / Main.

Luhmann, N. (1990): Der medizinische Code, in: Ders., Soziologische Aufklärung 5, Konstruktivistische Perspektiven, Opladen, S. 183-195.

Lupton, D. (1997): Consumerism, Reflexivity and the Medical Encounter, in: Social science and medicine 45, S.373-381.

Lutfey, K. (2005): On practices of 'good doctoring': reconsidering the relationship between provider roles and patient adherence, in: Sociology of health and illness 27, S.421-447.

Marstedt, G. (2003): Auf der Suche nach gesundheitlicher Information und Beratung: Befunde zum Wandel der Patientenrolle, in: J. Böcken / B. Braun / M. Schnee (Hrsg.), Gesundheitsmonitor 2003. Die ambulante Versorgung aus Sicht von Bevölkerung und Ärzteschaft, Gütersloh, S. 117-135.

Meisel, A. / M. Kuczewski (1996): Legal and ethical myths about informed consent, in: Archives of internal medicine 156, S. 2521-2526.

Meyer, J.W. / B. Rowan (1977): Institutionalized organizations: Formal structure as myth and ceremony, in: American journal of sociology, S. 340-363.

Meredith, D.B. (1996): Public health, preventive medicine and social services, 6. Auflage, London. 
Methoden-Report Patientenbeteiligung (2007), abgerufen am 11.11.2008 unter www.versorgungsleitlinie.de.

Miller, T. / M. Boulton (2007): Changing constructions of informed consent: Qualitative research and complex social worlds, in: Social science and medicine 65, S. 2187-2198.

Mojon-Azzi, S. / U. Wagner / D.St. Mojon (2003): Wie informiert ist der ophthalmologische Patient?, in: Klinische Monatsblätter Augenheilkunde 219, S.487-493.

Nettleton, S. / G. Hanlon (2006): ,Pathways to the doctor' in the information age: the role of ICTs in comtemporary lay referral systems, in: A. Webster (Hrsg.), New technologies in health care, Houndsmills, S. 57-70.

Noack, T. (2004): Eingriffe in das Selbstbestimmungsrecht des Patienten: Juristische Entscheidungen. Politik und ärztliche Positionen 1890-1960, Frankfurt / Main.

O'Donnell, M. / B. Monz / S. Hunskaar (2007): General preferences for involvement in treatment decision making among European women with urinary incontinence, in: Social science and medicine 64, S. 1914-1924.

Parsons, T. (1951): The Social System, New York - London.

Patient als Partner. Förderprogramm chronische Krankheiten und Patientenorientierung, abgerufen am 27.05.2007 unter http://patient-als-partner.de/index.php?article_id=42\&clang $=0$.

Reibnitz, C. von / P.-E. Schnabel / K. Hurrelmann (2001): Der mündige Patient. Konzepte zur Patientenberatung und Konsumentensouveränität im Gesundheitswesen, Weinheim.

Richards, T. (1998): Partnership with patients. Patients want more than simply information; they need involvement too, in: British medical journal 316, S.85-86.

Richter-Hebel, F. (1999):Warum kommen Patienten nicht wieder? Eine Praxis-Untersuchung, in: Zeitschrift für Allgemeinmedizin 75, S. 389-392.

Ridder, D. de / M. Depla / P. Severens / M. Malsch (1997): Beliefs on coping with illness: A consumer's perspective, in: Social science and medicine 44, S. 553-559.

Sabisch, K. (2007): Das Weib als Versuchsperson: medizinische Menschenexperimente im 19. Jahrhundert am Beispiel der Syphilisforschung, Bielefeld.

Salentin, K. (2004): Ziehen sich Migranten in , ethnische Kolonien’ zurück?, in: K.J. Bade / M. Bommes / R. Münz (Hrsg.), Migrationsreport 2004, Frankfurt / Main - New York, S. 97-116.

Scheibler, F. (2004): Shared decision making: von der Compliance zur partnerschaftlichen Entscheidungsfindung, Bern.

Scheibler, F. / U. Schwantes / M. Kampmann / H. Pfaff (2005): Shared decision-making, in: GGW (Wissenschaftliches Institut der AOK (WIdO) (Hrsg.): Gesundheit und Gesellschaft. Wissenschaft) $1 /$ 2005, S. 23-31.

Sillence, E. / P. Briggs / P.R. Harris / L. Fishwick (2007): How do patients evaluate and make use of online health information?, in: Social science and medicine 64, S. 1853-1862.

Stollberg, Gunnar (2001): Medizinsoziologie, Bielefeld.

Strauss, A. / S. Fagerhaugh / B. Suczek / C. Wiener (1980): Gefühlsarbeit. Ein Beitrag zur Arbeits- und Berufssoziologie, in: KZfSS 32, S. 629-651.

Streeck, Wolfgang (2005): Nach dem Korporatismus: Neue Eliten, neue Konflikte, MPIfG Working Paper 4 / 2005, Köln.

Street, R.L. / E. Krupat / R.A. Bell / R.L. Kravitz / P. Haidet (2003): Beliefs about control in the physicianpatient relationship, in: Journal of general internal medicine 18, S. 609-616.

Tacke, V. / G. Wagner (2005): Die Publikumsrolle des Kunden und die Semantik der Kundenorientierung. Eine differenzierungstheoretische Analyse, in: H. Jacobsen / S. Voswinkel (Hrsg.), Der Kunde in der Dienstleistungsbeziehung. Beiträge zur Soziologie der Dienstleistung, Wiesbaden, S. 127-148.

Tuckett, D. / M. Boulton / C. Olson (1985): A new approach to the measurement of patients' understanding of what they are told in medical consultations, in: Journal of health and aocial behavior 26, S. $27 \mathrm{ff}$. 
Vogd, W. (2002): Professionalisierungsschub oder Auflösung ärztlicher Autonomie: Die Bedeutung von evidence based medicine und der neuen funktionalen Eliten in der Medizin aus system- und interaktionstheoretischer Perspektive, in: Zeitschrift für Soziologie 31, S. 294-315.

Vogd, W. (2004): Ärztliche Entscheidungsprozesse des Krankenhauses im Spannungsfeld von Systemund Zweckrationalität. Eine qualitativ rekonstruktive Studie unter dem besonderen Blickwinkel von Rahmen (,frames“) und Rahmungsprozessen, Berlin.

Vogd, W. / I. Saake (2008): Einleitung: Moderne Mythen der Medizin. Eine organisationssoziologische Perspektive, in: W. Vogd / I. Saake (Hrsg.), Moderne Mythen der Medizin. Studien zur organisierten Krankenbehandlung, Wiesbaden, S. 7-38.

Weßels, B. (2000): Die Entwicklung des deutschen Korporatismus, in: Aus Politik und Zeitgeschichte, B 26-27.

Willems, D. (1992): Susan's breathlessness - The construction of professionals and laypersons', in: Lachmund, J. / G. Stollberg (Hrsg.), The social construction of illness. Illness and medical knowledge in past and present, Stuttgart, S. 105-114.

Williams, S.J. / M. Calnan (1996): Modern medicine. Lay perspectives and experiences, London.

Prof. Dr. Gunnar Stollberg

Fakultät für Soziologie

Universität Bielefeld

33501 Bielefeld

gunnar.stollberg@uni-bielefeld.de 